\title{
Alteraciones en la Erupción de Caninos Permanentes
}

\author{
Canine Permanent Eruption Alterations
}

\author{
"María Antonieta Pérez Flores; **Pablo Pérez Flores \& *** Claudia Fierro Monti
}

PEREZ, F. M. A.; PÉREZ, F. P. \& FIERRO, M. C. Alteraciones en la erupción de caninos permanentes. Int. J. Morphol., 27(1):139$143,2009$.

RESUMEN: En este trabajo son descritas alteraciones en la erupción de los caninos permanentes, su etiología, diagnóstico temprano y posibilidades terapéuticas. El canino recoge todos los problemas de espacio que puedan existir en la arcada y es frecuente la erupción en una zona alta. La inclusión de los caninos tiene una etiología compleja, favorecida por factores evolutivos, anatómicos y mecánicos. La impactación mandibular es menos frecuente y su incidencia se describe como veinte veces menos que la impactación del canino maxilar. La erupción ectópica de los caninos maxilares puede estar asociada a la reabsorción de las raíces de los incisivos adyacentes. A la edad de 8 a 10 años empieza a palparse la prominencia del canino en el fondo del vestíbulo. Los métodos radiográficos más utilizados son las radiografías panorámica, oclusal y periapical, con técnicas de tubo vertical y otras angulaciones. En la actualidad, es de gran ayuda la tomografía computarizada. El término tratamiento temprano implica una terapéutica en estadíos precoces de desarrollo, cuando la patología puede ser interceptada o detenida y los tratamientos más utilizados son exodoncia de caninos temporales y extracción seriada. En conclusión, las alteraciones en la erupción de caninos permanentes requiere conocimiento de los parámetros de normalidad, cronología y secuencia, junto a la aplicación de pruebas complementarias, para poder efectuar una evaluación temprana y pertinente y evitar las complicaciones derivadas de un diente impactado.

PALABRAS CLAVE: Erupción ectópica; Canino.

\section{INTRODUCCIÓN}

Después de los terceros molares, los caninos son los dientes que presentan mayor anomalías en su erupción, quedando atrapados en el hueso (Camilleri \& Scerri, 2003).

El concepto de anomalías en la erupción puede ir desde la erupción ectópica, erupción en otro lugar; hasta la inclusión dentro del hueso, con o sin impactación en alguna estructura (Canut, 2000; Chatem, 2004). La impactación vestibular suele estar asociada a problemas de espacio, pudiendo ser corregidos al hacerlo suficiente. La impactación palatina se asocia con alteraciones del trayecto. Es un problema amplio con una prevalencia de 1,7\% (Kurol, 2002). Tiene una etiología compleja, favorecida por factores evolutivos, anatómicos y mecánicos (Peñarrocha et al., 2003). No es un fenómeno nuevo y se han encontrado cráneos fechados con más de 4 milenios que presentaban esta patología (Otto, 2003). Un diagnóstico temprano y un seguimiento cuidadoso del desarrollo de la dentición son esenciales para la intervención temprana y la corrección de las anomalías eruptivas (Kurol).
El propósito de este trabajo es, por medio de una revisión bibliográfica, describir las posibles alteraciones en la erupción de los caninos permanentes, así como también, determinar sus posibles etiologías, diagnóstico temprano y posibilidades terapéuticas oportunas.

1. Trayectoria normal de erupción del canino permanente. Las características anatómicas del canino superior lo convierten en el diente con mayor potencial de supervivencia en la arcada. Desde un punto de vista funcional, los caninos son considerados dientes clave en la oclusión. Con un gran protagonismo estético, dan armonía al frente anterior, la línea de la sonrisa y surco geniano (Canut). Entre los 5 y 15 años de edad se ha observado que los caninos recorren al menos $22 \mathrm{~mm}$, durante este tiempo. En el plano lateral, los caninos muestran un movimiento significativo en dirección bucal entre los 10 y 12 años de edad. Antes de esta edad, tienen una dirección palatina. Aproximadamente 3/4 de la raíz se encuentra formada antes de la erupción y se completa 2 años después de ésta.

\footnotetext{
* Cirujano Dentista Magíster en Odontopediatría, Facultad de Odontología, Universidad de Concepción, Chile.

*** Médico Cirujano Ciclo de Destinación.

*** Especialista en Odontopediatría, Facultad de Odontología, Universidad de Concepción, Chile.
} 
Los caninos recogen todos los problemas de espacio que puedan existir en la arcada. (Canut; Kurol; McSherry, 1998).

\section{Anomalías de erupción en los caninos.}

Impactación. Los caninos quedan bloqueados en el espesor del hueso maxilar; cavidad nasal, órbita o la pared anterior del seno maxilar, pudiendo ser uni o bilateral y suele ocurrir en los caninos superiores, en la zona palatina, por detrás de las raíces de los incisivos (Ngan et al., 2005; Chaushu et al., 2003). Una sola etiología no es suficiente para causar la impactación o para permitir la diferencia de localización, vestibular o palatina. Existen estudios que certifican que el $85 \%$ de los caninos impactados palatinamente tenían espacio en el arco para erupcionar (Ngan et al.). Cuando un incisivo central se encuentra impactado por obstrucción u otra causa no específica, existe un 41,3\% de probabilidades que el canino ipsilateral esté desplazado (Chaushu et al.). La impactación de caninos tiene una incidencia que va desde 0,8 a 3,58\% (Aydin et al., 2004). La impactación mandibular se describe como veinte veces menos que la maxilar (Kindelan \& Cook, 1998). La incidencia de transmigración, o migración de la línea media del canino es mucho menos frecuente. Aunque la mayoría de los casos descritos en la literatura son unilaterales, la transmigración también puede ser bilateral (Aydin et al.). Un 60 a $80 \%$ de las transposiciones del canino superior son en palatino (Ericson \& Kurol, 1986) de un 15 a $30 \%$ en vestibular, 5 a $20 \%$ en posición intermedia. También puede quedar incluido en el seno maxilar o en el lado contralateral, en mandíbula generalmente se ubican en medio de la sínfisis mentoniana (Rebellato \& Schabel, 2003).

Transposición. Muchos dientes permanentes, especialmente caninos maxilares, segundos premolares y terceros molares o supernumerarios, pueden migrar y erupcionar en una posición ectópica. También es posible que el canino mandibular se invierta y migre a la línea media, lo que se denomina trasmigración del canino mandibular (Nakano et al., 2008; Camilleri \& Scerri; Costello et al., 1996). Aunque esta alteración ocurre sólo en caninos mandibulares, existe un caso en la literatura de canino maxilar que se atribuye principalmente a la corta longitud de las raíces de los incisivos (Aydin et al.). Se ha demostrado que la inervación pulpar de estos dientes proviene del lado opuesto (Costello et al.). La etiología de la transmigración no ha sido aún definida, se ha sugerido que puede tener determinantes genéticos y que respondería a una aberración del desarrollo (Aydin et al.; Mitchell, 1993). Podrían estar implicados fenómenos como: tumores, quistes, odontomas, fracturas, dientes supernumerarios o retención del canino temporal (Camilleri \& Scerri).

3. Consecuencias de las anomalías de erupción del canino. La mayor consecuencia es la mal oclusión que ge- nera, ya sea por la ausencia clínica del canino o por la alteración producida en otras piezas dentarias o a los tejidos adyacentes.

Reabsorción de Incisivos. La erupción ectópica de los caninos puede estar asociada a la reabsorción de las raíces de los incisivos adyacentes (Ericson \& Kurol, 1986); puede ser silenciosa, devastadora y repentina, llegando hasta comprometer la viabilidad del diente. Un 12,5\% de los caninos ectópicos causan reabsorciones y con el uso de las tomografías, se ha duplicado su diagnóstico (Otto; Heimisdottir et al., 2005). Las reabsorciones no se descubren clínicamente si no hasta los 10 años aproximadamente. Se ha observado que existe una preponderancia en el sexo femenino (Becker \& Chaushu, 2005). La reabsorción puede ocurrir en un 50\% de los casos de los incisivos adyacentes y $2 / 3$ de éstos ocurren con compromiso pulpar (Kurol). En algunos casos, estos dientes pueden sobrevivir, incluso, con grandes reabsorciones (Becker \& Chaushu).

Anquilosis. Los dientes impactados tienen el potencial de anquilosarse al hueso, causando todas las patologías asociadas a esta alteración (Rebellato \& Schabel).

Reabsorción idiopática. Por algún motivo, puede reabsorberse la raíz en parte o su totalidad de cualquier diente impactado (Canut).

4. Evaluación temprana. Desde los 8 a 10 años de edad empieza a palparse la prominencia del canino en el fondo del vestíbulo (Canut; Kurol; Ericson \& Kurol, 1986; Ericson \& Kurol, 1988). Si no se palpa a los 10 años, está indicado realizar un estudio radiológico para valorar su posición y la reabsorción radicular del canino temporal (Kindelan \& Cook). Si el canino permanente se observa en una radiografía frontal al nivel de la mitad distal de la corona del incisivo lateral, es de mejor pronóstico (Warford et al., 2003). Si se evalúa que existe una discrepancia óseo-dentaria, será necesario valorar la expansión maxilar. La evaluación clínica temprana debería comprender: El espacio en el arco para el canino no erupcionado, la morfología y la posición de los dientes adyacentes, el contorno del hueso, la movilidad del diente y un diagnóstico radiográfico para determinar la posición del canino: raíz, ápice, corona y longitud axial (Kurol).

El clínico debería sospechar de caninos incluidos en dos momentos: antes de los 10 años si existen antecedentes familiares de caninos impactados y si el paciente tiene incisivos pequeños, conoides o agenesias (impactación palatina); y después de los 10 años si hay asimetría en la palpación de la eminencia canina, si no se palpan los caninos o si hay inclinación del lateral hacia distal, aunque no se asocie necesariamente con patología. (Jacobs, 2000). 


\section{Técnicas diagnósticas}

Examen Clínico e Historia Clínica. El diagnóstico de un canino retenido debe hacerse en forma temprana. Se basa en observación, palpación, pruebas y antecedentes familiares. El signo clínico más evidente es la ausencia del canino permanente y suele ser asintomático.

En una primera visita se pueden observar algunos de estos signos clínicos (Lucea, 2005):

- Diastema, entre el incisivo lateral definitivo y el primer premolar.

- Persistencia del canino temporal en un niño de más de catorce años.

- Ausencia del abombamiento de la cortical a nivel del espacio de erupción.

- Desplazamiento de los dientes adyacentes en forma de abanico.

- Complicaciones infecciosas como fístulas o rara vez una celulitis geniana.

- Tinción de los dientes adyacentes por necrosis pulpar, consecuente a la rizálisis.

Examen radiográfico. Los métodos más utilizados son:

Radiografías periapicales: Informan en el plano frontal en sentido mesiodistal (Ericson \& Kurol, 1986). Con la técnica del "paralelismo" de Clark o "del objeto bucal", se puede diferenciar si el canino se encuentra en palatino o vestibular. (Peñarrocha et al.; Jacobs).

Radiografía panorámica: Informa la presencia de inclusión dentaria y su relación con las estructuras adyacentes, la patología asociada, dirección del canino. La ubicación en vestibular o palatino se puede determinar mediante el índice canino-incisivo (ancho del canino y del incisivo central homolateral) e índice canino-canino (ancho de canino con respecto al canino contralateral) (Peñarrocha et al.).

Radiografías oclusales: Dan información sobre la ubicación ántero posterior. Se utilizan dos métodos: a) Simpson, que consiste en haz de rayos perpendicular a la placa y paralelo al eje de los incisivos; y b) Belot en el que el haz de rayos no es perpendicular. El primero es más exacto (Peñarrocha $e t$ $a l$.). En el maxilar, se prefiere la técnica del paralelismo (Jacobs).

Combinación de radiografía oclusal y panorámica. (Jacobs).

Tomografía computarizada. Actualmente, es la técnica más útil para observar la trayectoria tridimensional del canino impactado. Ha duplicado el diagnóstico y hallazgos de reabsorción (Ericson \& Kurol, 2000). Informa la presencia o ausencia del canino, tamaño del folículo, inclinación de su eje axial, posición vestibular o palatina, la cantidad de hueso que lo cubre, situación y reabsorción de las piezas vecinas, consideraciones anatómicas locales y estado de desarrollo (Sawamura et al., 2003; Schramm et al., 2005).

\section{Pautas de intervención}

Exodoncia del canino temporal. Se considera una terapéutica temprana de elección en anomalías eruptivas de caninos permanentes en la arcada superior (Ericson \& Kurol, 1988). El canino permanente debe tener $2 / 3$ de la raíz para quitar el temporal y no retrase su erupción normal. Se recomienda realizar este procedimiento entre los 10 y 13 años y cuando la trayectoria del canino sea por palatino (Ericson \& Kurol, 1986; McSherry). Casi un $80 \%$ de caninos impactados en el maxilar erupcionan después de 12 meses de la extracción; si no ocurre o se hiciese un diagnóstico tardío, si existiera apiñamiento, reabsorción severa de las raíces de los incisivos laterales, o una trayectoria demasiado horizontal; se debe plantear un tratamiento correctivo (Ericson \& Kurol 1988; McSherry).

Extracción seriada. Consiste en una o más exodoncias de dientes temporales en dentición mixta y después de dientes permanentes, para crear una erupción favorable (Salzmann, 1949). Hay que considerar la posibilidad de autocorrección y que la extracción seriada extingue esta posibilidad y requiere un compromiso de parte del paciente (Salzmann, 1966; Taylor, 1971). En la mayoría de los casos, resultante de la inclinación del canino, se puede corregir el apiñamiento (Yoshihara et al., 2000).

Mantenedores de espacio. En pérdida prematura del canino temporal es necesario mantener el espacio para el canino permanente. La exfoliación prematura de caninos por la erupción de los incisivos laterales causa una constricción en la arcada porque no aumenta la distancia intercanina (Salzmann, 1949; Lucea).

Frenectomía del frenillo labial superior. Zetaplastía a los 7 años de edad, en los casos de que exista diastema interincisivo por inserción baja del frenillo labial superior, especialmente si existe una tendencia a la clase II (Lucea).

Eliminación de posibles interferencias. En la vía de erupción se pueden encontrar odontomas, supernumerarios, quistes, entre otros (Lucea). En el caso de transmigración de caninos mandibulares, un tratamiento temprano involucra extraer el canino impactado antes que se encuentre formada su raíz (Rebellato \& Schabel). 
En conclusión, la etiología de las alteraciones en caninos es muy diversa y está relacionada con la ubicación del canino en los huesos maxilar o mandibular. La consecuencia que adquiere mayor importancia es la reabsorción de las raíces de los incisivos. Esto se debe al compromiso dentario que esto significa. La evaluación temprana o la presunción de que algo interfiere con la erupción de los caninos, debe estar orientada a evitar que se impacte o se incluyan en los maxilares y para esto se utilizan técnicas diagnósticas que van desde un riguroso examen clínico y pruebas complementarias, como radiografías panorámicas, periapicales y oclusales, hasta el desarrollo de nuevos métodos de imagenología, como es la tomografía computarizada. Las opciones de tratamiento temprano son pocas e incluyen, en la mayoría de los casos, la extracción del canino deciduo.

El diagnóstico de las alteraciones de la erupción de caninos permanentes involucra conocimiento de los parámetros de normalidad, cronología, oportunidad y secuencia, junto con el desarrollo de pruebas complementarias, para efectuar una evaluación temprana y pertinente y así evitar las complicaciones derivadas de un diente impactado.

PEREZ, F. M. A.; PÉREZ, F. P. \& FIERRO, M. C. Canine permanent eruption alterations. Int. J. Morphol., 27(1):139-143, 2009.

SUMMARY: This study describes the alterations in the eruption of the permanent canines, etiology, early diagnosis and therapeutic possibilities. The canine one gathers all the problems of space that can exist in the arches and is frequent the eruption in a high zone. The inclusion of the canine has a complex etiology, favored by evolutionary, anatomical and mechanical factors. The mandibular impaction is less frequent and its incidence is described less like twenty times than the impaction of the maxilar canine one. The ectopic eruption of maxillary canines can be associated to the reabsorbing by the roots of the adjacent incisors. From 8 to 10 years begins to be noticed the prominence of the canine in the bottom of vestibule The most used radiographies methods are the panoramic x-ray, oclusals and periapicals with techniques of vertical tube and other angles. At the present time it is helpful the computed tomography. The term "early treatment" implies a therapeutic action in early stages of development, when the pathology can be intercepted or stopped, and the most used are exodontias of temporary canines and serial extraction. In conclusion, the canine alterations of the eruption of permanent involve knowledge of the normality parameters, chronology and sequence, along with the development of complementary tests, to carry out an early and pertinent evaluation and to avoid the complications derived from an impacted tooth.

\section{KEY WORDS: Ectopic eruption; Canine.}

\section{REFERENCIAS BIBLIOGRÁFICAS}

Aydin, A.; Yilmaz, H. H. \& Yildrim, D. Incidence of canine impaction and transmigration in a patient population. Dentomaxillo. Rad., 33:164-9, 2004.

Becker, A. \& Chaushu, E. Long-term follow-up of severely resorbed maxillary incisor after resolution of an etiologically associated impacted canine. Am. J. Orthod. Dentofacial. Orthop., 127:650-4, 2005.

Canut, J. A. Ortodoncia Clínica y Terapeútica. $2^{\mathrm{a}}$ Edición. Barcelona, Ed Masson, 2000.

Camilleri, S. \& Scerri, E. Transmigration of a mandibular canines- A review of the literature and report of five cases. Angle Orthod., 73:753-62, 2003.

Chatem, R. Maxillary canine impaction; a final twist in the tale? J. Orthod., 31(1):13-4, 2004.

Chaushu, S.; Zilberman, Y. \& Becker, A. Maxillary incisor impaction and its relationship to canine displacement. Am. J. Orthod. Dentofacial. Orthop., 124:144-50, 2003.
Costello, J. P.; Worth, J. C. \& Jones, A. G. Transmigration of permanent mandibular canines. Br. Dent. J., 181:212-3, 1996.

Ericson, S. \& Kurol, J. Early treatment of palatally erupting maxillary canines by extraction of the primary canines. Eur. J. Orthod., 10(4):283-95, 1988.

Ericson, S. \& Kurol, J. Radiographic assessment of maxillary canine eruption in children with clinical signs of eruption disturbance. Eur. J. Orthod., 8(3):133-40, 1986.

Ericson, S. \& Kurol, J. Resorption of incisors after ectopic eruption of maxillary canines: a CT study. Angle Orthod., 70(6):415-23, 2000.

Heimisdottir, K.; Bosshardt, D. \& Ruf, S. Can the severity of root resorption be accurately judged by means of radiograhs? A case report with histology. Am. J. Orthod. Dentofacial Orthop., 128:106-9, 2005.

Jacobs, S. Radiographic localization of the unerupted teeth: 
Further findings about the vertical tube shift method and other localization techniques. Am. J. Orthod. Dentofacial Orthop., 118:439-47, 2000.

Kindelan, J. \& Cook, P. The ectopic maxillary canine: A case report. Br. J. Orthod., 25:179-80, 1998.

Kurol, J. Early treatment of tooth-eruption disturbances. Am. J. Orthod. Dentofacial Orthop., 121(6):588-91, 2002.

Lucea, A. Caninos incluidos. Tratamiento con biomecánica de arcos dobles. Ortodoncia Clínica, 8(1):22-32, 2005.

McSherry, B. The ectopic maxillary canine: A review. British J. Orthod., 25:209-16, 1998.

Mitchell, L. Displacement of a mandibular canine following fracture of the mandibule. Br. Dent. J., 174:417-8, 1993.

Nakano, Y.; Shibahara, T.; Sekiguchi, H. \& Yakushiji, M. Transmigration of impated mandibular canine to opposite side. Pediatric Dental J., 18(1):70-3, 2008.

Ngan, P.; Hornbrook, R. \& Weaver, B. Early timely management of ectopically erupting maxillary canines. Semin. Orthod., 11:152-62, 2005.

Otto, R. Early and unusual incisor resorption due to impacted maxillary canines. Am. J. Orthod. Dentofacial Orthop., 124:446-9, 2003.

Peñarrocha, M. A.; Peñarrocha, M.; Larrazábal, C. \& García, B. Caninos incluidos, consideraciones quirúrgicas y ortodóncicas. Arch. Odontoestomatol., 19(5):336-46, 2003.

Rebellato, J. \& Schabel, B. Treatment of a patient with an impacted transmigrant mandibular canine and palatally impacted maxillary canine. Angle Orthod., 73:328-36, 2003.

Salzmann, J. A. Serial extraction as a corrective procedure in dental orthopaedic therapy. Am. J. Orthodontic., 35(6):471-6, 1949.

Salzmann, J. A. Serial Extraction in general dental practice. Am. J. Orthodontic., 52(2):145-6, 1966.

Sawamura, T.; Minowa, K. \& Nakamura, M. Impacted teeth in the maxilla: usefulness of 3D dental-CT for preoperative evaluation. Eur. J. Orthod., 47:221-6, 2003.
Schramm, A.; Rücker, M.; Sakkas, N.; Schön, R.; Düker, J. \& Gellrich, N. C. The use of cone beam CT in craniomaxilo facial surgery. Int. Congress Series, 1281:12004,2005

Taylor, R. Controlled serial extraction. Am. J. Orthodontic., 60(6):567-99, 1971.

Warford, J.; Grandhi, R. \& Tira, D. Prediction of maxillary canine impaction using sectors and angular measurement. Am. J. Orthod. Dentofacial Orthop., 124:651-5, 2003.

Yoshihara, T.; Matsumoto, Y.; Suzuki, J.; Sato, N. \& Oguchi, H. Effect of serial extraction alone on crowdindg: Spontaneous changes indentation after serial extraction. Am. J. Orthod. Dentofacial Orthop., 118:611-6, 2000.

\author{
Dirección para correspondencia: \\ Dra. $M^{a}$ Antonieta Pérez \\ Dpto. Pediatría Bucal \\ Universidad de Concepción \\ Av. Roosvelt 1550. \\ Casilla 160-C \\ Concepción - CHILE \\ Fono 56-41-2204292 \\ Fono Fax 56-41-2243311 \\ Email mperezf@udec.cl \\ Recibido : 08-11-2008 \\ Aceptado: 10-12-2008
}


